\title{
Gestes et prosodie dans la parole aphasique non fluente
}

\author{
Gaëlle Ferré \\ LLING - UMr 6310, Chemin de la Censive du Tertre, BP 81227, 44312 Nantes cedex 3 \\ Gaelle.Ferreduniv-nantes.fr
}

\section{RÉSUMÉ}

L'aphasie non-fluente se caractérise par de fréquentes recherches lexicales et un débit de parole beaucoup plus lent que la parole non aphasique. Pour les patients atteints de ce type d'aphasie, la communication avec leur entourage est de ce fait rendue difficile et se trouve souvent très altérée. Une des propositions thérapeutiques pour améliorer la qualité de vie de ces patients consiste à rééduquer les patients avec des alternatives plus multimodales. Cela suppose bien entendu que la gestualité soit une alternative possible pour les patients, et que leurs gestes ne soient pas affectés au même titre que leur parole. Cet article se propose donc d'étudier la gestualité de personnes aphasiques et de la comparer aux gestes produits par des personnes non aphasiques. Les résultats montrent que si l'on compare les gestes référentiels et non-référentiels, c'est essentiellement dans leur structure interne que les gestes des personnes aphasiques sont affectés, et que cette structure interne correspond aux troubles de la parole.

\section{ABSTRACT \\ Gesture and prosody in non-fluent aphasic speech.}

Non-fluent aphasia is characterized by frequent word searches and a slower speech-rate than is found in fluent speech. For people with aphasia (PWA), everyday communication is therefore very much impaired. Multimodal communication has been suggested as an alternative therapeutic treatment in order to improve patients' daily interactions. Such a treatment however presupposes that gesture is a possible alternative for patients, and that their gestures are not impacted by aphasia in the way their speech is. This paper therefore presents a study of PWA's gestures in a comparison with speakers without aphasia. Results show that PWA's referential and non-referential gestures are impacted essentially in their internal structure and that this structure matches PWA's speech impairment.

MOTS-CLÉS : Aphasie non fluente, prosodie, gestualité.

KEYWORDS: Non-fluent aphasia, prosody, gesture.

\section{Introduction}

L'aphasie est un trouble du langage entraîné par des lésions cérébrales, le plus souvent dues à un AVC, qui peut affecter la production linguistique écrite et orale et/ou la compréhension de la parole (Dipper et al., 2015). Les lésions neuronales affectent des fonctions linguistiques spécifiques, les troubles pouvant révéler des déficiences phonologiques, syntaxiques, lexicales et sémantiques (Preisig et al., 2015). La parole aphasique non-fluente se caractérise principalement par un effort de parole important lié à de fréquentes recherches lexicales, une articulation des sons difficile et une syntaxe 
basée essentiellement sur la production de groupes nominaux (Macauley \& Handley, 2005). Du fait des difficultés à communiquer qu'elle entraîne, l'aphasie a de lourdes conséquences psycho-sociales pour les personnes qui en sont atteintes (Blom Johansson, 2012; Nyström, 2006) :

- Perte d'emploi,

- Isolement des personnes aphasiques (PA) et dégradation des relations familiales et sociales,

- Perte d'estime de soi face à un sentiment d'incompétence linguistique pouvant mener à un état dépressif.

Afin d'améliorer la qualité de vie des patients, les thérapeutes et les chercheurs s'interrogent sur l'opportunité d'une thérapie multimodale (M-MAT : Multimodal Aphasia Therapy) en comparaison avec une thérapie centrée uniquement sur la parole (CIAT : Constraint-Induced Aphasia Therapy). Les résultats des études menées sur les progrès des patients ayant suivi l'une ou l'autre thérapie sont mitigés (Rose et al., 2015) : les effets d'une thérapie multimodale dépendent de la situation de communication et de la sévérité de l'aphasie des patients. L'efficacité d'une thérapie multimodale présuppose d'ailleurs que la gestualité des patients ne soit pas affectée au même titre que la parole et puisse venir compenser la parole déficiente ou au moins faciliter l'accès au lexique. Si les travaux sur la gestualité produite - spontanément ou à la demande des thérapeutes - par les personnes aphasiques (PA) se sont multipliés ces dix dernières années, nous sommes encore loin de connaître les similarités et différences entre la gestualité des PA et celle des personnes non aphasiques (PNA), ni le rôle potentiellement compensatoire ou facilitateur des gestes produits par les PA (Pritchard et al., 2015). Par ailleurs, les travaux existant se basent principalement sur des corpus de parole spécifiques qui peuvent avoir une grande influence sur la production gestuelle spontanée (narrations à partir d'images, production de discours procéduraux, ...), ou sur des études qualitatives de la production de gestes par les PA dans des corpus non contrôlés (interactions en milieu familial).

L'objectif de cet article est donc de présenter une étude prosodique de la gestualité des PA dans des récits personnels semi-contrôlés en comparaison avec les gestes produits par des PNA : la distribution gestuelle des PA est-elle semblable à celle des PNA, le nombre de gestes et leur structure interne sont-ils similaires chez les deux groupes de locuteurs?

\section{Contexte théorique}

Chez les aphasiques de Broca, les mots produits en position initiale de groupe sont plus longs que les mots produits en position finale à l'inverse du rythme de parole non aphasique (Danly \& Shapiro, 1982) et les syllabes non-finales sont aussi plus longues que les syllabes finales (Louis, 2003). Les PA non fluentes montrent en particulier des difficultés à initier la production d'un mot (Kurowski \& Blumstein, 2016), avec des erreurs phonologiques plus fréquentes en début de mot (Tuller, 1984). Les PA non fluentes souffrent également de nombreuses paraphasies : métathèses, épenthèses, substitutions et suppressions de son(s) dans les mots, assimilations, confusions de mots qui engendrent de nombreuses auto-corrections.

Les travaux menés sur la gestualité des PA trouvent parfois des résultats contradictoires. Selon Cicone et al. (1979) et Feyereisen (1983), les PA non fluentes ne sont pas plus fluentes en gestualité qu'elles ne le sont dans la parole. Elles produisent moins de gestes que les non-aphasiques, le débit 
de parole étant directement corrélé à la fréquence gestuelle et au rapport geste/mot. D'autres travaux trouvent au contraire que la plupart des PA ont tendance à utiliser la communication non verbale pour compenser la parole déficiente (Hogrefe et al., 2013; de Beer et al., 2017). Cependant, parmi les travaux qui relatent une compensation possible de la parole par la gestualité, les résultats sont également contradictoires. Alors que pour Smith (1987) citée dans (Ahlsén, 1991), les PA les plus sévèrement atteintes compensent plus que les PA moins sévèrement atteintes, Mol et al. (2013) trouvent au contraire que les PA les plus sévèrement atteintes gestualisent moins facilement que les PNA pour compenser la parole. Ces différences dans les résultats obtenus pour la densité gestuelle des PA s'expliquent en grande partie cependant par le fait que les calculs n'ont pas été menés sur les mêmes unités de parole (Cocks et al., 2013) : alors que certaines études choisissent le rapport geste/mot ou la proposition syntaxique pour calculer le nombre de gestes produits par les PA, d'autres choisissent une unité temporelle (nb de gestes par seconde ou minute).

Enfin, il n'est pas encore certain du rôle joué par la gestualité chez les PA : si certains auteurs comme ceux déjà cités dans le paragraphe précédent estiment que la gestualité peut être utilisée par les PA pour compenser une parole déficiente, d'autres pensent que la gestualité facilite la récupération de la forme phonologique correcte des mots dans les recherches lexicales sans se substituer à la parole (de Ruiter, 2006), quand d'autres encore accordent les deux fonctions aux gestes dans cette population (Kroenke et al., 2013). Comme le remarque Ahlsén (2015), la gestualité linguistique et la parole sont étroitement liées, mais ont néanmoins aussi une certaine indépendance l'une par rapport à l'autre, ce qui expliquerait ces résultats en apparence contradictoires. Quant aux effets des thérapies multimodales, elles sont elles-aussi contrastées : si elles sont efficaces pour certains patients, elles ne le sont pas pour d'autres (Beeke et al., 2015).

\section{Questions de recherche}

La plupart des études mentionnées dans la section précédente ont examiné les relations gestes/parole dans des expériences basées sur des descriptions d'images ou d'actions qui encouragent la production de gestes iconiques ou de pointages, les autres types de gestes ayant été largement ignorés. Nous connaissons donc mal la production gestuelle des PA dans des environnements moins contraints en comparaison avec les gestes des PNA. C'est sur ce point que cet article se propose de porter : lorsqu'ils ne sont pas contraints par la nature iconique d'une réponse ou par son lien avec un support imagé, la gestualité des PA est-elle similaire à celle des PNA ? De plus, la structure interne des gestes produits par les PA est-elle semblable à celle des PNA? Par exemple, qu' advient-il des gestes lorsque les mots sont difficiles à exprimer? Sont-ils maintenus, répétés ou abandonnés?

\section{Données et méthodologie}

\subsection{Corpus}

Afin de répondre à ces questions, j'ai utilisé certains fichiers vidéos d'AphasiaBank (MacWhinney et al., 2011), une base de données multimédias qui rassemble des locuteurs aphasiques et non-aphasiques de plusieurs langues, enregistrés selon le même protocole. Les tâches du protocole 
incluent quatre genres : récits personnels élicités par des questions très similaires chez les PA et les PNA, descriptions d'images, récits de contes et discours procédural mais seuls les récits personnels ont été utilisés pour cette étude.

Dans la base de données, j'ai sélectionné 4 PA ( 2 femmes et 2 hommes) et 4 PNA ( 3 femmes et 1 homme), tous locuteurs d'anglais américain. Parmi les PA, 2 patients sont atteints d'une aphasie de Broca et les deux autres souffrent d'une aphasie transcorticale motrice. L'aphasie des quatre PA est modérée selon le score WAB (Western Aphasia Battery) qu'elles ont obtenu à une série de tests de compréhension et de production linguistique orale et écrite pratiqués en milieu hospitalier et répertoriés dans la base de données. Les informations concernant les quatre PA de l'étude sont données dans la Table 4.1.

\begin{tabular}{|c|c|c|c|c|c|c|}
\hline Participants & Sexe & Durée de l'aphasie & Date de l'enreg. & Age & Aphasie & Score WAB \\
\hline ACWT01a & F & 11 ans 8 mois & 2012 & 69,9 & Broca & 63,9 \\
ACWT02a & F & 3 ans 30 jours & 2012 & 53,1 & TCM & 74,6 \\
adler18a & H & 5 ans 75 jours & 2010 & 71,5 & TCM & 59,8 \\
elman03a & H & 11 ans & 2009 & 55,2 & Broca & 66,2 \\
\hline
\end{tabular}

TABLE 1 - Description des PA sélectionnées dans AphasiaBank pour l'étude (les informations concernant la durée de l'aphasie et l'âge du participant sont données en relation avec la date où l'enregistrement a eu lieu)

\subsection{Traitement des données}

La nature du corpus pose la question des unités choisies pour comparer de manière adéquate la parole des PA et des PNA, sachant que les unités prosodiques ou syntaxiques traditionnellement adoptées pour la transcription dans les études multimodales peuvent se révéler problématiques. Par exemple, les Unités Inter-Pausales sont difficiles à adopter ici du fait de la présence récurrente de pauses silencieuses longues - y compris parfois en milieu de mot - chez les PA lors de leurs fréquentes recherches lexicales. Ces pauses fréquentes et parfois assez longues, ainsi que la présence de nombreuses amorces de mots rendent également difficile la détection de groupes intonatifs. Enfin, l'agrammaticalité de la parole des aphasiques de Broca (notamment l'absence récurrente de verbes et la substitution de certains groupes syntaxiques par des onomatopées, par exemple) exclut une transcription des propositions syntaxiques. Sachant que le phonème n'est pas une unité adaptée à une analyse multimodale car elle n'a pas du tout la même granularité que le geste, c'est le groupe de souffle qui a été choisi ici pour une transcription verbatim du corpus sous PRAAT (Boersma \& Weenink, 2009). Chaque groupe de souffle était délimité par une reprise de souffle audible et/ou un changement de tour de parole. 224 groupes de souffle ont été annotés pour les PA contre 324 pour les PNA. Le corpus a ensuite été transcrit en mots également alignés avec le signal (694 mots pour les PA contre 2899 pour les PNA). Ces transcriptions incluaient les pauses silencieuses et remplies, ainsi que les amorces de mots, rires, etc. Dans une piste séparée, j'ai noté le nombre de syllabes dans chaque groupe de souffle, sans noter la transcription de chaque syllabe.

Avec le logiciel d'annotation des fichiers multimédias ELAN (Sloetjes \& Wittenburg, 2008), l'ensemble des gestes manuels produits par les PA et les PNA sur les 8 récits personnels ont été 
annotés (434 gestes pour les PA contre 291 pour les PNA, soit un total de 725 gestes). Les gestes ont été catégorisés en gestes référentiels (gestes qui font référence à un objet ou une action concrèt(e) ou à un concept abstrait) et en gestes non-référentiels (gestes qui ne font pas référence à un objet, une action ou un concept, comme par exemple, les gestes liés à la recherche lexicale, les gestes qui expriment une modalité ou qui sont liés à l'organisation du discours, ainsi que les battements). Au total, les PNA ont produit 132 gestes référentiels contre 159 gestes non-référentiels, et les PA 164 gestes référentiels contre 270 gestes non-référentiels.

Enfin, toujours sous ELAN, les différentes phases gestuelles (Kendon, 2004) ont été annotées. Parmi ces phases, on compte la préparation, c'est-à-dire la mise en place des mains pour la réalisation du geste. La phase de réalisation est la partie communicative du geste, et la rétraction correspond au relâchement des mains (ici, soit accompagné d'un retrait total vers la position de repos, e.g. les mains posées sur la table, soit accompagné d'un retrait partiel, e.g. relâchement des mains sans retrait total). Deux autres phases ont également été notées : une possible tenue pré-réalisation ou post-réalisation pendant lesquelles la/les main(s) marque(nt) une pause avant ou après la phase de réalisation, mais en conservant la configuration manuelle établie pendant la préparation ou pendant la réalisation.

\section{Résultats}

Afin de répondre aux questions posées dans la section 3, les différences entre les PA et les PNA ont été testées par Modèles Mixtes Linéaires Généralisés (GLMM) (Bates et al., 2014) avec le programme d'analyses statistiques R 3.4.0 (R Core Team, 2012). Les locuteurs ont été inclus comme facteur aléatoire pour rendre compte de la variabilité inter-locuteurs.

Comme l'on pouvait s'y attendre, on observe une différence significative entre les participants (PA vs. PNA) sur la durée des groupes de souffle $(\beta=1061.4, \mathrm{SE}=431.2, p<0.05)$, avec une durée moyenne de $4029.7 \mathrm{~ms}$ pour les PA et de $2839 \mathrm{~ms}$ pour les PNA. On observe aussi une différence significative entre les deux groupes de participants pour ce qui est de la vitesse d'articulation (nb de syll/sec dans les groupes de souffle sans compter les temps de pause) avec en moyenne $2.5 \mathrm{syll} / \mathrm{sec}$ pour les PA contre $4.1 \mathrm{syll} / \mathrm{sec}$ pour les PNA $(\beta=-2.14, \mathrm{SE}=0.28$,

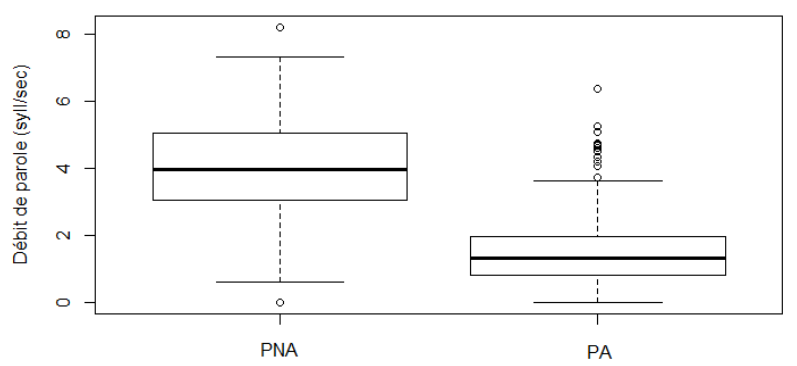

FIGURE 1 - Débit de parole moyen des PNA et des PA dans les groupes de souffle $p<0.001$ ). Le débit de parole (nb de syll/sec en comptant les pauses) est lui-aussi significativement différent entre les deux groupes $(\beta=-2.3, \mathrm{SE}=$ $0.24, p<0.001$ ) avec un débit moyen de $1.5 \mathrm{syll} / \mathrm{sec}$ pour les PA contre $3.8 \mathrm{syll} / \mathrm{sec}$ pour les PNA. Cette différence de débit n'est cependant pas liée à un nombre plus élevé de pauses remplies au sein des groupes de souffle pour les $\mathrm{PA}(\beta=0.63, \mathrm{SE}=0.29, p=0.27)$ ou de pauses silencieuses $(\beta=$ $1.40, \mathrm{SE}=0.33, p=0.14)$.

Si la durée des pauses remplies n'est pas non plus significativement différente entre les deux groupes 
$(\beta=-65.4, \mathrm{SE}=32.4, p=0.09)$, avec une durée moyenne de $584.8 \mathrm{~ms}$ pour les pauses remplies des PA contre 513.4 ms pour les PNA, c'est en revanche la durée des pauses silencieuses au sein des groupes de souffle qui est significativement différente entre les deux populations $(\beta=-290.5, \mathrm{SE}=$ $83.5, p=0.01$ ), avec une durée moyenne des pauses silencieuses de $682.4 \mathrm{~ms}$ pour les PA contre $371.7 \mathrm{~ms}$ pour les PNA.

En ce qui concerne la densité gestuelle (nombre de gestes par seconde tous types de gestes confondus), on n'observe pas de différence significative entre les deux groupes $(\beta=0.16, \mathrm{SE}=$ $0.07, p=0.06$ ) avec une densité gestuelle moyenne de 0.38 gestes/sec pour les PA contre 0.24 gestes/sec pour les PNA. La différence entre les deux groupes reste valable si l'on prend en compte le nombre de gestes en fonction du temps d'articulation $(\beta=0.35, \mathrm{SE}=0.06, p<0.005)$. Cette absence de significativité pour la densité gestuelle s'applique de la même manière aux gestes non-référentiels $(\beta=0.102, \mathrm{SE}=0.05, p=0.10)$ et référentiels $(\beta=0.06, \mathrm{SE}=0.05, p=0.24)$. Sur le nombre total de gestes produits, le rapport geste/mot est donc de 0.6 pour les PA contre 0.1 pour les PNA. Si l'on ne compte pas les amorces de gestes et les gestes liés à la recherche lexicale, la proportion reste la même entre les deux groupes avec 0.5 geste/mot pour les PA contre 0.09 geste/mot pour les PNA. Il n'y a pas non plus de différence significative sur la durée des gestes entre les PA et les PNA $(\beta=-98.7, \mathrm{SE}=188.4, p=0.61)$, que ce soit pour les gestes référentiels $(\beta=433, \mathrm{SE}=342, p=0.25)$ ou les gestes non-référentiels $(\beta=-363.1, \mathrm{SE}=227.8, p=0.16)$.

Les principales différences entre les PA et les PNA se situent dans la structure interne des gestes, dans certaines phases gestuelles. En ce qui concerne la préparation, la différence de durée de cette phase n'est pas significativement différente entre les deux groupes $(\beta=120.8, \mathrm{SE}=54, p=0.06)$. En revanche, les PA produisent significativement plus de préparations que les PNA $(\beta=0.7, \mathrm{SE}=0.23$, $p=0.01$ ). Pour ce qui est de la tenue pré-réalisation, les PA ne produisent pas plus de tenues que les PNA ( $\beta=1.4, \mathrm{SE}=0.6, p=0.08)$, mais lorsqu'ils en produisent, ces phases sont significativement plus longues que pour les PNA $(\beta=79.8, \mathrm{SE}=30.4, p=0.03)$. Les phases de réalisation ne sont pas d'une durée significativement différente entre les deux groupes $(\beta=-12.4, \mathrm{SE}=58.3, p=0.83)$, mais elles sont moins nombreuses chez les PA que chez les PNA $(\beta=-2.9, \mathrm{SE}=6, p=0.02)$. Les phases de tenues post-réalisation ne sont ni plus nombreuses dans un groupe que dans un autre $(\beta=-0.07$, $\mathrm{SE}=0.32, p=0.8)$, ni plus longues $(\beta=-243.7, \mathrm{SE}=170, p=0.2)$. La même observation peut être faite pour les rétractions qui ne sont ni plus nombreuses dans un groupe que dans l'autre $(\beta=-0.29$, $\mathrm{SE}=0.42, p=0.5)$, ni plus longues $(\beta=-49.7, \mathrm{SE}=54.9, p=0.4)$.

L'ensemble de ces résultats est représenté dans la Figure 2 qui présente la durée moyenne des phases gestuelles et leur pourcentage en fonction du nombre total de gestes produit par chaque groupe. L'absence de significativité sur la durée des tenues post-réalisation s'explique sans doute par la grande variabilité de ces tenues chez les PA, alors que l'absence de significativité du nombre de pré-réalisation entre les deux groupes s'explique par le très petit nombre de ce type de phase chez les PNA, ce qui est en soi révélateur.

\section{Discussion et conclusion}

Cette étude portait sur les liens entre parole et gestualité chez des personnes aphasiques non fluentes $(\mathrm{N}=4)$ en comparaison avec des personnes non-aphasiques $(\mathrm{N}=4)$, à partir d'un corpus 

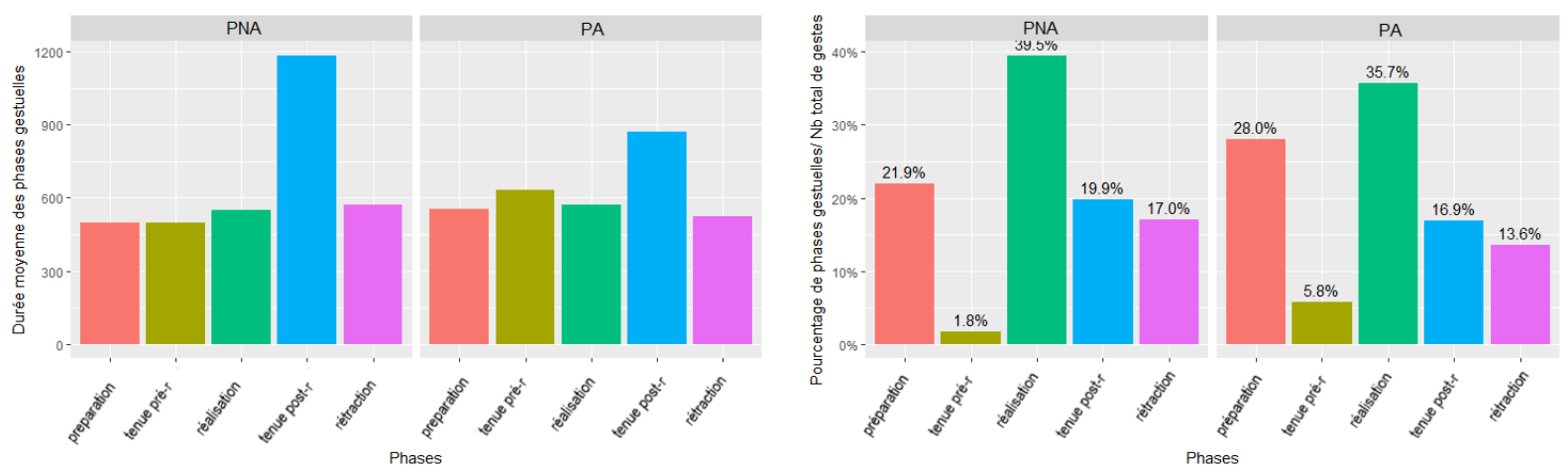

FIGURE 2 - Durée moyennes des phases gestuelles (gauche) et pourcentages d'occurrence des phases gestuelles en fonction du nombre total de gestes (droite) chez les PNA et les PA

d'enregistrements vidéos fourni par AphasiaBank (MacWhinney et al., 2011). L'étude portait principalement sur des récits personnels, e.g. des situations d'interaction où les PA sont certes contraintes par les questions posées par le chercheur, mais dans lesquelles la parole ne peut s'appuyer sur des images présentes dans le contexte d'interaction, ni sur l'iconicité du discours procédural.

Comme l'on pouvait s'y attendre, les résultats montrent que le débit de parole des PA et leur vitesse d'articulation sont beaucoup plus lents que celui des PNA, et que ceci est dû non seulement à des difficultés d'articulation des mots et de nombreuses amorces au niveau verbal, mais aussi à la présence de pauses silencieuses plus longues que chez les PNA, mais pas nécessairement plus nombreuses. Les pauses remplies ne sont pas significativement différentes dans les deux groupes de participants. Comme l'a montré Swerts (1998), les pauses remplies jouent essentiellement un rôle dans la structuration du discours contrairement aux pauses silencieuses qui permettent de gérer des difficultés plus locales et sont en phase avec les nombreuses recherches lexicales que peut engendrer l'aphasie.

En ce qui concerne les gestes, la densité gestuelle (nb de gestes par seconde) n'est pas différente dans les deux groupes de locuteurs, que ce soit pour les gestes référentiels et les gestes non-référentiels. Mais bien entendu, comme le débit de parole des PA est lent que celui des PNA, cela signifie que les PA produisent plus de gestes par nombre de mots que les PNA ce qui confirme les études de Feyereisen (1983) et Cocks et al. (2013). Ces résultats sont également compatibles avec ceux des travaux qui trouvent que les PA gestualisent plus que les PNA, et qui sont basés principalement sur le rapport des gestes au nombre de mots. Dans une recherche à venir, ces résultats seront affinés par une étude des types de gestes au sein des catégories un peu larges que sont les gestes référentiels et non-référentiels, avec des pistes prometteuses concernant l'utilisation des battements et des gestes conventionnels par les PA dans ce type d'interaction.

Enfin, on observe des différences de structure interne des gestes entre les deux groupes de participants. Les PA produisent proportionnellement moins de phases de réalisation que les PNA et ceci montre que leur gestualité est liée à leurs difficultés en parole : de même que les PA produisent beaucoup plus d'amorces de mots que les PNA, ils ont plus d'amorces de gestes. Il leur arrive de préparer un geste puis de rétracter les mains sans que ce geste ait été réalisé. On peut donc dire que les abandons gestuels vont de pair avec les amorces verbales. Les PA produisent aussi plus de phases 
de préparation que les PNA et là encore, ce schéma correspond à leur production verbale, certains PA s'exprimant principalement sous la forme de groupes nominaux sans liens syntaxiques entre les groupes, leurs gestes sont moins enchaînés. Enfin, la durée plus longue de leurs tenues pré-réalisation montre également que les PA font des pauses plus longues avant de réaliser leurs gestes jusqu'à ce que soit résolues les recherches lexicales. Ceci correspond à ce qui avait déjà été observé dans le bégaiement par Mayberry \& Jaques (2000), à savoir que le geste est suspendu - plutôt qu'abandonné - jusqu'à ce que le mot puisse être produit quand la syllabe initiale est plus longue. Il serait d'ailleurs intéressant de voir sur un plus grand nombre de locuteurs si la structure des gestes est identique dans l'aphasie de Broca et l'aphasie transcorticale motrice.

\section{Remerciements}

Je tiens à remercier B. MacWhinney et D. Fromm pour m'avoir donné accès à AphasiaBank, ainsi que tous les locuteurs qui ont participé à cette base de données. AphasiaBank fournit des informations précieuses sur la parole aphasique en mettant à la disposition des chercheurs des enregistrements multimodaux par ailleurs difficiles à collecter. Je remercie aussi deux relecteurs anonymes pour leurs commentaires constructifs sur une version précédente de cet article.

\section{Références}

Ahlsén E. (1991). Body Communication as Compensation for Speech in a Wernicke's Aphasic-a Longitudinal Study. Journal of Communication Disorders, 24, 1-12.

AhlsÉn E. (2015). Gestures Used in Word Search Episodes - by Persons with and without Aphasia. In K. JoKInEN \& M. Vels, Eds., The 2nd European and the 5th Nordic Symposium on Multimodal Communication, p. 9-15, Tartu, Estonie.

BATES D., ET AL. (2014). Linear mixed-effects models using eigen and s4. Computer program : http ://cran.r-project.org.

BEEKE S., ET AL. (2015). Conversation focused aphasia therapy : investigating the adoption of strategies by people with agrammatism. Aphasiology, 29(3), 355-377.

Blom Johansson M. (2012). Aphasia and Communication in Everyday Life : Experiences of persons with aphasia, significant others, and speech-language pathologists. Phd, Department of Public Health and Caring Sciences, Uppsala.

Boersma P. \& WeEninK D. (2009). Praat : doing phonetics by computer (Version 5.1.05) Computer program : http ://www.fon.hum.uva.nl/praat/.

CiCOne M., ET AL. (1979). The Relation between Gesture and Language in Aphasic Communication. Brain and Language, 8, 324-349.

CoCKS N., ET AL. (2013). The impact of impaired semantic knowledge on spontaneous iconic gesture production. Aphasiology, 27(9), 1050-1069.

DANly M. \& Shapiro B. (1982). Speech Prosody in Broca's Aphasia. Brain and Language, 16, 171-190.

DE BeER C., ET AL. (2017). How Much Information Do People With Aphasia Convey via Gesture? American Journal of Speech-Language Pathology, 26, 483-497. 
DE RUiter J. P. (2006). Can gesticulation help aphasic people speak, or rather, communicate? International Journal of Speech-Language Pathology, 8(2), 124-127.

DIPPER L., ET AL. (2015). The language-gesture connection : Evidence from aphasia. Clinical Linguistics \& Phonetics, 29(8-10), 748-763.

FEYEREISEN P. (1983). Manual Activity During Speaking in Aphasic Subjects. International Journal of Psychology, 18, 545-556.

HOGREFE K., ET AL. (2013). Gestural expression in narrations of aphasic speakers : redundant or complementary to the spoken expression? In TIGER, p. 1-4, Lund, Suède.

Kendon A. (2004). Gesture. Visible Action as Utterance. Cambridge : Cambridge University Press.

KroEnKe K.-M., ET AL. (2013). Lexical learning in mild aphasia : Gesture benefit depends on patholinguistic profile and lesion pattern. Cortex, 49(10), 2637-2649.

KuRowski K. \& Blumstein S. E. (2016). Phonetic basis of phonemic paraphasias in aphasia: Evidence for cascading activation. Cortex, 75, 193-203.

Louis M. (2003). Etude longitudinale de la dysprosodie d'un cas d'Aphasie Progressive Primaire : analyse des variables temporelles. Thèse de doctorat, Université d'Aix en Provence.

Macauley B. L. \& Handley C. L. (2005). Gestures Produced by Patient With Aphasia and Ideomotor Apraxia. Contemporary Issues in Communication Science and Disorders, 32, 30-37.

MacWhinney B., ET AL. (2011). AphasiaBank : Methods for Studying Discourse. Aphasiology, 25(11), 1286-1307.

MAYBERRY R. I. \& JAQUES J. (2000). Gesture production during stuttered speech : insights into the nature of gesture-speech integration. In D. MCNEILL, Ed., Language and Gesture, Language, Culture \& Cognition, p. 199-214. Cambridge : Cambridge University Press.

MOL L., ET AL. (2013). Gesturing by speakers with aphasia : how does it compare? Journal of Speech and Hearing Research, 56(4), 1224-1236.

NystRöm M. (2006). Aphasia - an existential loneliness : A study on the loss of the world of symbols. International Journal of Qualitative Studies on Health and Well-being, 1(1), 38-49.

Preisig B. C., ET AL. (2015). Perception of co-speech gestures in aphasic patients : A visual exploration study during the observation of dyadic conversations. Cortex, 64, 157-168.

PRITCHARD M., ET AL. (2015). Language and iconic gesture use in procedural discourse by speakers with aphasia. Aphasiology, 29(7), 826-844.

R CORE TEAM (2012). A language and environment for statistical computing. $R$ foundation for statistical computing. Computer program : http ://www.r-project.org.

Rose M. L., ET AL. (2015). Comparing multi-modality and constraint-induced treatment for aphasia : a preliminary investigation of generalisation to discourse. Aphasiology, 30(6), 678-698.

Sloetjes H. \& WitTenburg P. (2008). Annotation by category - ELAN and ISO DCR. In 6th International Conference on Language Resources and Evaluation (LREC 2008), Marrakech, Maroc. SwerTs M. (1998). Filled Pauses as Markers of Discourse Structure. Journal of Pragmatics, 30, 485-496.

Tuller B. (1984). On Categorizing Aphasic Speech Errors. Neuropsychologia, 22(5), 547-557. 